\title{
Evaluación de la apropiación de las TIC, en la práctica docente del programa de ingeniería de sistemas de la Universidad Francisco de Paula Santander, Ocaña
}

\author{
Claudia Marcela Duran-Chinchilla ${ }^{a} \&$ Alveiro Alonso Rosado-Gómez ${ }^{b}$ \\ ${ }^{a}$ Facultad de Educación Artes y Humanidades, Universidad Francisco de Paula Santander, Ocaña, Colombia.cmduranc@ufpso.edu.co \\ ${ }^{b}$ Facultad de Ingenierias, Universidad Francisco de Paula Santander, Ocaña, Colombia. aarosadog@ufpso.edu.co
}

\begin{abstract}
Resumen-Este artículo evaluó la apropiación de las TIC en la práctica docente dentro del programa de ingeniería de sistemas de la Universidad Francisco de Paula Santander. La investigación se abordó desde el enfoque cualitativo ya que a partir de él se podía conocer la apropiación de las TIC por parte de los docente; para ello de aplicó una entrevista en profundidad; los informantes clave fueron docentes de las áreas que fundamentan el currículo, las cuales son: ciencias básicas, ciencia básica de ingenierías, formación profesional y formación complementaria. Los resultados de la investigación permitieron determinar que los docentes no se han apropiado de las TIC y están en un periodo de transición en cuanto al uso de las mismas; factores como edad, formación disciplinar, tipo de contratación, nivel de escolaridad y su propia percepciones acerca de las TIC, influyen notoriamente en la apropiación de la tecnología dentro de la práctica docente.
\end{abstract}

Palabras Clave-TIC; apropiación de TIC; práctica docente.

Recibido: 11 de Julio de 2016. Revisado: 12 de octubre de 2016. Aceptado: 27 de octubre de 2016.

\section{Evaluation of the appropriation of ICT in the teacher practice in engineering systems program of the Francisco de Paula Santander University, Ocaña}

Abstract - This paper was evaluated the appropriation of ICT in teaching practice in the Systems Engineering program at the University Francisco de Paula Santander. The research was engaged from a qualitative approach, because from there you can interpret the teaching methods of teachers. For the research was applied an interview in depth; the key informants were teachers in the areas underlying the curriculum, which are the following: basic science, basic science of engineering, vocational training and further training. The research results allowed to determine that teachers do not have appropriated ICT yet, and the teachers are in a transition period for their use. Factors such as age, disciplinary trainning, kind of contract, education level and their own perceptions about ICT, has a remarkable impact on the appropriation of technology in teaching methods used.

Keywords - ICT; ICT appropriation; teaching practice.

\section{Introducción}

Las Tecnologías de Información y Comunicación (TIC), es un término comúnmente usado y ampliamente conocido que está cada vez más inmerso en la sociedad. Las TIC juegan un papel fundamental en la trasformación de la sociedad, tanta es su importancia que se considera como uno de los pilares de la economía global Eugenia [1,2] de los aspectos económicos, las TIC, juegan un papel fundamental en la formación de los profesionales, ya que son ellas, las que dan soporte a la información y a la vez se convierten en canales de comunicación. De tal manera que [3] indica que existe una relación simbólica entre las TIC y los flujos de información, comunicación y conocimiento que empleado con fines pedagógicos, como lo señala [4], pueden validar la correcta aplicación de las TIC, en el ambiente de aprendizaje; dado que la tecnología por sí sola no garantiza que pueda ser aplicada a la educación, lo que produce la necesidad de tener referentes que indiquen las posibles aplicaciones de una tecnología, es por esta razón que documentos como el informe Horizon, se convierten en fuentes de consulta para conocer el impacto de tecnologías actuales y futuras en el ámbito educativo [5].

El sistema educativo pasa una época de cambios generados por la introducción tecnológica y sobre todo por el desarrollo de las TIC, que han reformado las relaciones sociales y que a su vez intervienen en la relación tecnología - educación. Pese a que las tecnologías no son del todo nuevas, siguen siendo una innovación en las aulas de clase y su incorporación a la educación se convierte en un reto para los docentes [6]. Uno de los cambios mencionados afecta la práctica docente centrada en el docente, en cómo él trabaja, se expresa, se comporta, se relaciona, la metodología empleada en sus clases; en pocas palabras el uso de las tecnologías en el aula, está relacionada con la disposición y la habilidad del docente para usar las herramientas TIC. La forma en que se muestre, se desarrolle y se evalúen los contenidos, puede o no agradar al estudiante, quien es aquel que puede considerarse como el beneficiario o el afectado, por una buena o mala práctica académica [7].

Los procesos de enseñanza y aprendizaje han estado influidos por la incorporación de TIC como una forma de alcanzar los propósitos pedagógicos planteados y para estar a la par con las exigencias de la sociedad presente, la cual cada día demanda que en la formación de los profesionales se incluya el uso de las nuevas tecnologías, toda vez que estas se han convertido en un medio de comunicación dentro y fuera de las

Como citar este artículo: Duran-Chinchilla, C. M. \& Rosado-Gómez, A. A.. Evaluación de la apropiación de las TIC, en la práctica docente del programa de Ingeniería de Sistemas de la Universidad Francisco de Paula Santander Ocaña. Revista Educación en Ingeniería 12 (23) 64-68, Febrero, 2017. 
empresa [8]. En tal sentido, éste paso ha generado cambios relevantes en los roles de cada uno de los individuos y organizaciones implicados en este proceso: los alumnos, los docentes y el personal administrativo. En el caso de los docentes, estos deben enfrentarse a retos que implican tener la mente abierta para adaptarse y acomodarse a los cambios en la nueva sociedad del conocimiento y a la era de la globalización económica y tecnológica, por lo que la educación universitaria está llamada a formar profesionales capaces de adaptarse a los rápidos cambios sociales $[9,10]$.

Se puede percibir, entonces las TIC no solo como herramienta técnica, sino también como instrumento cultural que permite reconocer el funcionamiento de la sociedad, generando cambios en el conocimiento, la enseñanza y el aprendizaje. Los docentes entonces, están llamados a aportar y desarrollar competencias en el uso de las TIC, para que la educación universitaria mejore debe estar en sintonía con las novedades en el proceso pedagógico en tres componentes: política educativa, administración de la institución y desarrollo profesoral [11].

Así mismo, el Plan Nacional Decenal de Educación en su interés por cubrir estas necesidades de la implementación de tecnologías en la educación, propone enmarcar los procesos pedagógicos con el uso de las TIC, independientemente del contenido curricular que se esté impartiendo, con el ánimo de centrar la enseñanza en el estudiante [12]. Para lograr la integración de las TIC con el currículo, se crea la necesidad de un análisis conceptual que justifique esta inmersión; porque de nada sirve integrar las TIC en la práctica pedagógica de los docentes, si ésta no se enriquece desde la misma didáctica de la enseñanza [13]. Para integrar curricularmente a las TIC, se debe engranar su aplicación en función de estimular el aprendizaje de los estudiantes en un determinado contexto; una buena práctica pedagógica mediada por el uso de las TIC, implica incorporar estos dos elementos como un todo y no como elementos complementarios. Para lograr este propósito se propone que los docentes deben dominar aspectos tecnológicos que aborden nuevos escenarios didácticos, que estimulen al estudiante en su aprendizaje, por encima de la tecnología como tal; es decir los docentes deben preocuparse menos por los aspectos propios de la ofimática y centrar su atención en los recursos robustos que faciliten y medien la enseñanza [4].

Este contexto indica la relevancia que tiene en la aplicación de las TIC en la educación, el dominio y la sensibilidad que los docentes tengan sobre ella; por esta razón éste estudio tuvo como propósito establecer las apropiaciones que los docentes de ingeniería de sistemas de la Universidad Francisco de Paula Santander Ocaña tienen de las TIC en su práctica pedagógica; el estudio partió de un investigación anterior, en cuanto a la incorporación de las TIC por parte de los docentes de la Facultad de Ingeniería como medio para mejorar la calidad educativa, en el cual se determinó que los docentes de ingeniería si usan las TIC en sus clases, pero la concepción que se tiene de ellas es más como una herramienta de apoyo en las clases y no como verdaderamente una herramienta de acercamiento al mundo cultural, social, económico y político que exige el medio globalizante en el cual estamos inmersos; igualmente se reconoció que integran las TIC esporádicamente en los currículos; también se pudo establecer que los docentes de Ingeniería de Sistemas hacen mayor uso de las TIC en relación a los docentes de Ingeniería Civil y Mecánica.

Desde esa mirada es importante detallar la apropiación de las TIC por parte de los docentes de ingeniería de sistemas. En primer lugar es una política institucional la inmersión de las TIC en los currículos y en las prácticas educativas, y en segundo lugar porque el plan de estudios de ingeniería de sistemas en su proceso de autoevaluación, determinó utilizar el b-learning, como estrategia de fomento al trabajo independiente por parte de los estudiantes; esto produce como consecuencia que los docentes que orientan las asignaturas del programa, deban tener competencias en el manejo de las TIC, para construir e interactuar con los recursos y actividades que están disponibles dentro de la plataforma de aprendizaje de la universidad y que deben estar enmarcadas dentro de los lineamientos pedagógicos, comunicacionales y tecnológicos establecidos por la universidad. Esto produjo adelantar esfuerzos para identificar el grado de apropiación que tienen los docentes en la aplicación de las TIC, con fines educativos y de esta forma comenzar un proceso de capacitación en donde el docente con pueda mejorar sus competencias en cuanto a apropiación de TIC, hasta estar en la capacidad de proponer sus propios recursos y actividades.

\section{Metodología}

Para el desarrollo del estudio se tuvo en cuanta el enfoque cualitativo, ya que éste, tal y como lo plantea [14], trata de hacer una análisis como un todo e identificar las razones del comportamiento manifestado. En lo que relacionado con el nivel de investigación se abordó la investigación descriptiva, ya que ésta busca identificar características relevantes de grupos o comunidades a partir de un objeto de estudio, en este caso las TIC [15].

En lo que respecta a la recolección de información se diseñó una entrevista en profundidad, dado que este tipo de instrumento permite interactuar y ahondar a través del diálogo con cada uno de los informantes; en el diseño del instrumento se tuvo en cuenta dos categorías: la primera categoría apropiación de TIC, y la segunda uso de TIC, cada una de ellas compuesta por subcategorías de estudio como aprestamiento de TIC, integración curricular TIC, herramientas TIC, incorporación TIC. La validación del instrumento se llevó a cabo a través del juicio de expertos el cual es entendido como una validación que hace personas que dominan y tienen reconocimiento en el tema, para que estos a su vez emitan un juicio sobre las respuestas suministradas en las entrevistas [16].

Los informantes clave, estuvieron conformados por docentes del programa de Ingeniería de Sistemas, se tomó un docente por cada área establecida en el currículo del programa: ciencias básicas, ciencia básica de ingenierías, formación profesional y formación complementaria, el criterio para la selección de los informantes fue el de docentes de género femenino y masculino, distintas edades y que fuesen ingenieros en sistemas, además se tuvo en cuenta el tipo de vinculación docente, es decir catedráticos, ocasionales y tiempo completo. 


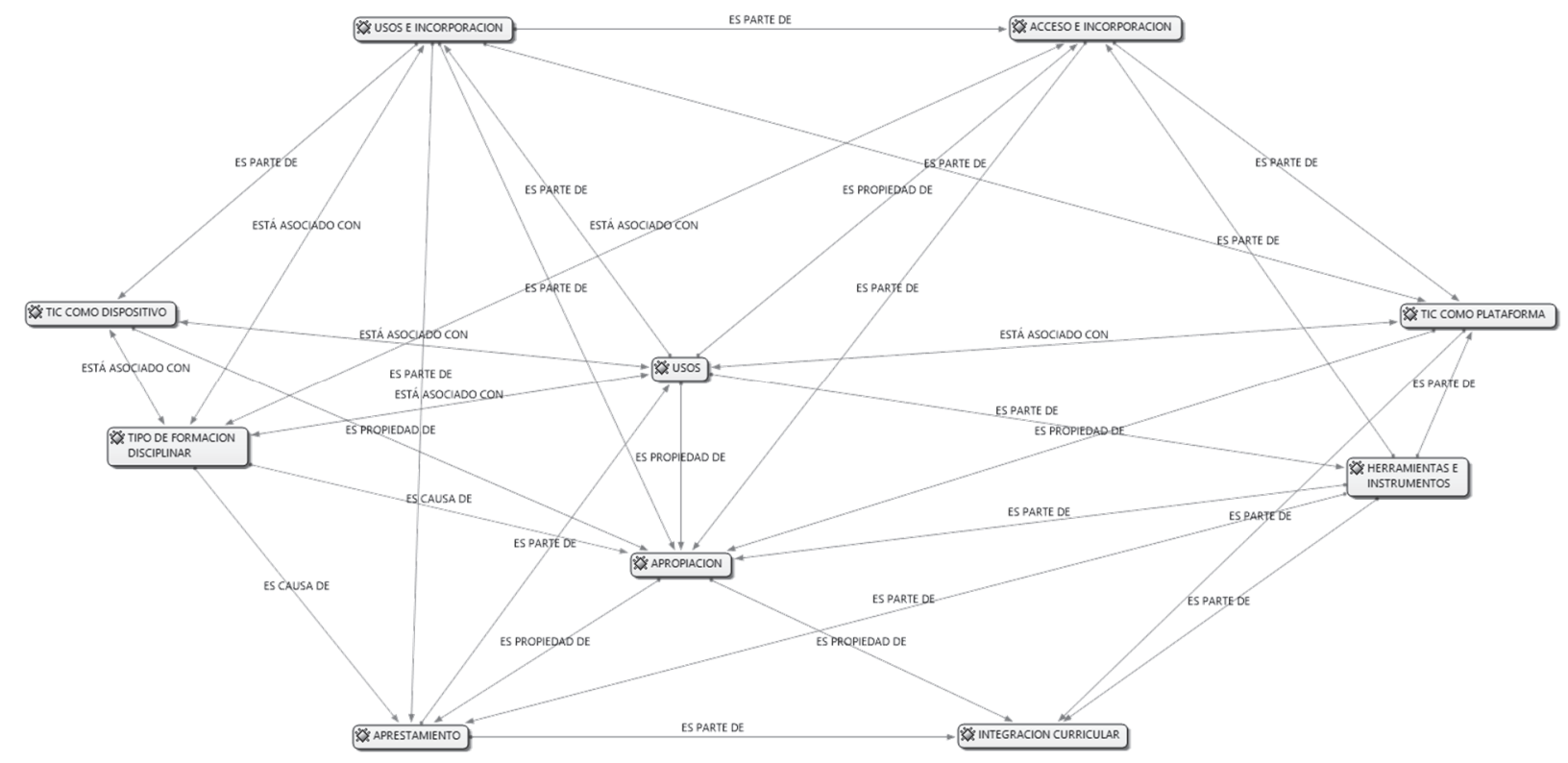

Figura 1. Gráfica de relaciones

Fuente: Autores del proyecto

La información socavada se analizó aplicando el software Atlas TI, esta herramienta permitió establecer unidades hermenéuticas y relaciones entre categorías y subcategorías.

\section{Resultados}

La apropiación de TIC, se fundamenta en el uso positivo y acertado de las TIC con respecto a la construcción de conocimiento, valores, innovación y toda relación estructural del conocimiento [17], esto involucra incluir no sólo el conocimiento de estas herramientas y de su potencialidad, sino también su uso normal en los distintos campos pedagógicos, culturales y sociales de cada individuo [18]. Por ello, se realizó una exploración en torno a la apropiación que los docentes del programa de Ingeniería de Sistemas de la Universidad Francisco de Paula Santander Ocaña tienen en cuanto a las TIC.

Para establecer las relaciones en cuanto a apropiación TIC, en la Tabla 1, se muestran las categorías y subcategorías establecidas para evaluar la apropiación de las TIC en los docentes del plan de estudios de ingeniería de sistemas.

Cuanto a las categorías uso y apropiación de TIC. Se puede establecer que las herramientas de mayor afinidad de algunos docentes entrevistados, es el computador y el internet como medio de consulta, las dos herramientas, manifiestan los docentes, son necesarios en su vida cotidiana para comunicarse con sus alumnos, familiares y compañeros de trabajo. Por ejemplo, el uso del internet ha beneficiado en ellos el uso de canales comunicativos como Facebook, Twitter y Skype en donde mantienen una constante comunicación con todo su entorno académico de forma online, esto en el caso de los docentes del área de ciencias básicas de ingeniería $\mathrm{y}$ formación profesional.

Partiendo de la realización de las entrevistas, la investigación arrojó los siguientes resultados, los cuales están soportados a través de la Fig. 1, se indica las relaciones en Tabla 1

Categorías

\begin{tabular}{ccc}
\hline Objetivo & Categoría & Subcategoría \\
\hline Evaluar los & & Aspectos \\
procesos de & Apropiación de TIC & demográficos \\
apropiación de las & Uso significativos de las & Aprestamiento de TIC \\
TIC que & tecnologías de & en la práctica docente \\
evidencian los & informáticas y de & Integración curricular \\
docentes del & comunicación, en el cual & de TIC en las practica \\
programa de & se ejerce un grado de & docente \\
ingeniería de & control y elección sobre la & Incorporación TIC en \\
sistemas de la & tecnología y los & el aula \\
Universidad & contenidos, pudiéndose & Herramientas TIC \\
Francisco de & considerar al uso útil, & utilizada por los \\
Paula Santander & fructífero, valioso e & docentes en el aula y \\
Seccional Ocaña & importante para el usuario & fue ella como \\
en sus prácticas & & \\
docente & & \\
\hline Evaluar el nivel & & \\
de uso que & Uso TIC & \\
evidencian los & Variación notable en la & \\
docentes del & sociedad y a la larga un & Uso de TIC como \\
programa de & cambio en la educación, & dispositivo \\
Ingeniería de & en las relaciones & Uso de TIC como \\
Sistemas de la & interpersonales y en la & plataforma \\
Universidad & forma de difundir y & \\
Francisco de & generar conocimientos & \\
Paula Santander & & \\
Seccional Ocaña & & \\
\hline Fuente Autores del proyecto &
\end{tabular}

Fuente. Autores del proyecto

También, se logró evidenciar que los docentes recurren al correo electrónico para la administración y distribución de información institucional y personal. Igualmente utilizan el Video Beam para la proyección multimedia de contenidos de las asignaturas, sin embargo, manifiestan que la falta de recursos en la Institución es notable y que usar este tipo de herramientas es un proceso complejo.

Como se ve, se le concede un alto grado de importancia a las herramientas TIC para el proceso enseñanza - aprendizaje, pues según las opiniones de los docentes, a través del uso de las herramientas como software interactivo, diapositivas, plataforma Moodle, etc., han conseguido reducir los índices de atención 
dispersa de algunos estudiantes, falta de escucha y falta de comprensión de temas álgidos en las asignaturas.

Otros docentes, especialmente los del área de ciencias básicas y formación complementaria manifiestan que no encuentran la manera práctica y metodológica de incorporar las herramientas en sus clases, debido a que pocas veces han necesitado de ellas para llevar a cabo el desarrollo de su asignatura. Se puede afirmar entonces que algunos docentes prestan poca atención a la inclusión de las TIC en su labor pedagógica, mostrando así, temores y dificultades para lograr integrarlas en el aula. Este mismo grupo de docentes asegura que pese a que tienen, por ejemplo, celulares de última tecnología, emplean aplicaciones de mensajería instantánea en sus dispositivos móviles como son WhatsApp e Instagram, pero solo como uso personal y que no ven la utilidad de los mismos como recurso pedagógico.

Para los dos grupos de docentes: los que ven en las TIC una herramienta importante en el proceso de aprendizaje y los que no ven en ellas un instrumento indispensable en la práctica docente, se puede asumir que el concepto que se tiene de las TIC es como dispositivo (Video Beam, computador, celular, etc.) y como plataforma (Internet, Moodle, base de datos o software), en los dos casos se entendería que en lugar de apropiación sólo se indica aprestamiento dado que algunos docentes usan algunos recursos las TIC en sus clases y fuera de ellas, sin tener claro la utilidad que ellas prestan dentro del aula; es decir, el valor de las TIC como recurso y herramienta pedagógica, didáctica, curricular $\mathrm{y}$ metodológico no es preciso y clara.

En lo que respecta a la edad, los resultados dejan percibir que la brecha entre edad y uso de TIC resulta ser notable; dado que a mayor sea la edad del docente menos familiaridad tiene con la tecnología [19]. Esto quiere decir, que a mayor edad menos posibilidad de integrar las TIC a los procesos de enseñanza aprendizaje [20].

En lo que respecta a la relación tipo de vinculación docente (catedrático, ocasional y tiempo completo), la apropiación de TIC, está asociada con el tiempo de dedicación docente en este caso los docentes catedráticos solo acuden a las clases asignadas y el tiempo de dedicación es restringida y está sujeta a múltiples situaciones dado que algunos docentes tienen labores que atender fuera de la adquirida con la institución, mientras que los docentes de planta su dedicación es exclusiva a la universidad y por su puesto a la academia, lo cual produce que su interés por integrar más elementos a sus clases que beneficien el aprendizaje.

En cuanto a la integración curricular de las TIC, es evidente que se debe tener una intención claro para integrar las TIC en los procesos académicos y curriculares pues no se trata en la utilización de las TIC como un instrumento más dentro del aula [13,21]. Desde esa mirada, se puede indicar que, aunque algunos docentes integran de alguna forma curricularmente las TIC en sus clases; no lo hacen con apropiación, pues la integración curricular de TIC se entiende como el proceso de hacerlas completamente parte del currículo, permeándolas con los principios educativos y la didáctica que conforman el mecanismo del aprender. Ello esencialmente exige un uso integral y eficaz para un propósito del aprender específico en un dominio o una disciplina curricular.

En lo relacionado con la formación disciplinar de los docentes, se puede afirmar que en cierta medida que sí existe relación en cuanto al uso de TIC y el área disciplinar, pues los entrevistados manifiestan que la incorporación de las TIC depende en gran medida de la disciplina, pues creen que los ingenieros en sistemas deben tener mayor manejo de las herramientas TIC que los docentes que asumen asignaturas como ética, matemáticas o todas las relacionadas con el área complementaria, factor que lleva a pensar que las percepciones que se tiene de las TIC no es abierta, y equivoca, pareciera que se asumen las TIC solo como dispositivos o como el simple manejo de algunas herramientas que ayudan a realizar clases más amenas y no como verdaderamente un medio de acercamiento $y \mathrm{de}$ reconocimiento cultural, social, político y económico del contexto.

\section{Discusión}

Entre los elementos que afectan el uso de las TIC en la práctica docente está las representaciones que algunos docentes han constituido acerca de estas herramientas, las cuales son los elementos que presentan como argumentos para no utilizarlas dentro de su práctica educativa.

En el caso particular de esta investigación, a través de las entrevistas a los docentes del programa de Ingeniería de Sistemas, se puede apreciar que la incorporación de las TIC, en el desarrollo curricular obedece a una necesidad de estar en sintonía con lo que el medio o los estudiantes solicitan, más que a un empoderamiento o apropiación de las mismas; pareciera que simplemente se queda en el uso de algunas herramientas y no como un proceso cognitivo que explote toda su potencial en el beneficio del estudiante con elementos y recursos didácticos adecuados a la temática expuesta dentro del curso [22].

Desde las apreciaciones de los informantes, se puede inferir que los docentes no han implementado las TIC en sus currículos y solo las usan de acuerdo a la necesidad; hay que tener en cuenta que el uso de las TIC no se puede hacer como un medio para desarrollar una clase dinámica e "innovadora" [13], sino más bien se debe tratar de producir nuevas formas de producción de conocimiento a través del uso de las mismas.

Es posible también repensar en cuanto a las aptitudes de los docentes, especialmente en el campo de la formación universitaria, ya que las TIC deben incorporarse, como se ha venido planteando, en la programación curricular y al quehacer docente, con el fin de que el uso de las herramientas tecnológicas favorezcan el proceso de enseñanza/aprendizaje [4].

De tal manera, las capacidades docentes deben incluir la capacidad para determinar, reflexionar y debatir, tomar decisiones, intervenir y evaluar la práctica, así como poseer un conocimiento técnico sobre recursos tecnológicos [8], y no que se quede en solo el uso de las mismas sin un propósito claro, únicamente con la intención de apoyarse de algunas herramientas, lo que queda en apresamiento, sin ir más allá de lo que exige la apropiación de las herramientas tecnológicas de información y comunicación [4].

Por otro lado, quizás que los docentes manifiesten interés y motivación por educarse en las TIC, pero su uso como herramienta educativa es restringido y con poco rango de aplicaciones. Su uso se focaliza sustancialmente a proyectos personales, la mayoría utiliza las computadoras para tareas de bajo nivel tales como registro de notas, bases de datos, buscar información en Internet y casualmente para proyectar una clase en Power Point con proyector de multimedia. Sin embargo y pese a cualquier obstáculo que se presente para hacer efectivo el uso y apropiación de las TIC, se hace indispensable que el docente entienda sus potencialidades y restricciones, utilizando los recursos adecuados con fines pedagógicos [21]. 


\section{Conclusión}

La apropiación de las TIC, está reducido en este caso por los niveles o grados de uso que los docentes tengan de las mismas. La apropiación permite a los docentes, en este caso, hacer suyas las herramientas tecnológicas para emplearlas en su relación con los escenarios educativos, socioculturales y contextos de actividad en los que se desenvuelve. La apropiación tecnológica es la manera en que los sujetos asumen las herramientas, basados en la interpretación y proximidad que tiene cada uno con éstas, construyendo así una realidad subjetiva. De tal forma que es importante tener en cuenta que la formación de los docentes debe comprender tanto el conocimiento de las posibilidades que las TIC les brindan como las distintas metodologías y didácticas que se implemente en los procesos de enseñanza-aprendizaje.

Así mismo, fue común encontrar en esta investigación que, aunque unos docentes hacen un esfuerzo por ajustarse a las TIC, prefieren las prácticas tradicionales, por lo cual siguen prefiriendo la interacción directa con los estudiantes. Algunos profesores encuentran una explicación de este fenómeno en lo acelerado que se ha dado el cambio que han generado estas herramientas y la necesidad de aplicación en el espacio universitario.

La utilización de las TIC por parte de los docentes del plan de estudios de ingeniería de sistemas, está sujeta a un cambio progresivo en la medida en que la persona conciba positivamente los beneficios que ofrecen las distintas herramientas y va interactuando con ellas hasta apropiarlas, para luego hacer partícipes a los demás actores sociales de las posibilidades que éstas pueden ofrecer.

A pesar que la utilización de las TIC dentro del programa de Ingeniería de Sistemas, en el área de formación profesional hace parte del currículo y por ende se tiene que aplicar la tecnología dentro de los cursos; esta no se aplica con fines didácticos ni pedagógicos, solo como un complemento a los conceptos teóricos que especifica el currículo, motivo por el cual se puede decir que dentro del programa existe un uso significativo, pero poca apropiación.

\section{Referencias}

[1] Eugeni, K., Tecnología de la información y comunicación en la formación docente, UNESCO Guía de Planificación. [En líena]. Paris, 2004. [Consultado: junio 27 de 2016]. Disponible en: http://unesdoc.unesco.org/images/0012/001295/129533s.pdf

[2] The World Bank, Measuring knowledge in the world's economies, knowledge for development program, World Bank Institute, Washington, 2008.

[3] Torrent, J. y Ficapal, P., TIC, conocimiento, redes y trabajo, Barcelona: Editorial OUC, 2009.

[4] Cabero, J., Incidentes críticos en la incorporación de la TIC en las universidades, de Congreso Nacional EDUTEC. Gestión de las Tecnologías de la Comunicación e Información, 2002.

[5] Johnson, L., Adams-Becker, S., Estrada, V. and Freeman, A., Horizon Report: 2014 Higher Education Edition, Austin, 2014.

[6] Gros-Salvat, B., Sancho-Vinuesa, T., Borges-Sáiz, F., Bautista, G., García-González, I., López-Pérez, C., Mas-García X. y Lara-Navarra, P., Evolución y retos de la educación virtual construyendo el e-learning del siglo XXI, Barcelona: UOC, 2011.

[7] Briones, G., Hacia una pedagogía del conocimiento, Bogotá: Mac Graw Hill, 1999.

[8] Ferreiro, R. y de Napoli, A., Más allá del salón de clases: Los nuevos ambientes de aprendizajes, Revista Complutense de Educación, pp. 333-346, 2008.

[9] Cobo-Romaní, J.C., El concepto de tecnologías de la información. Benchmarking sobre las definiciones de las TIC en la sociedad del conocimiento, Zer, 14(27), pp. 295-318, 2009.
[10] Chupitaz, L., García M. y Sánchez, D., Informática aplicada a los procesos de enseñanza aprendizaje, Lima: Fondo Editorial de la Pontificia Universidad Católica de Perú, 2005.

[11] UNESCO, Estándares para competencias en TIC para docentes, Londres: UNESCO, 2008.

[12] MEN, Compendio General PNDE, Bogotá: Ministerio de Educación Nacional, 2006.

[13] Sanchez-LLabaca, J., Integración curricular de las TICs: Conceptos e ideas, pp. 2-29, 2012.

[14] Martinez-Miguelez, M., La investigación cualitativa, Revista IIPS. 9, pp. 123-146, 2009.

[15] Cazau, P., Introducción a la investigación en ciencias sociales, Buenos Aires: Tercera Edición, 2006.

[16] Escobar-Pérez J. y Cuervo-Martínez, A., Validez de contenido y juicio de expertos: Una aproximación a su utilización, Avances en Medición. 6. [en línea]. Tomado http://www.humanas.unal.edu.co/psicometria/files/7113/8574/5708/Art iculo3_Juicio_de_expertos_27-36.pd junio 28 de 2016, pp. 27-36, 2008

[17] Afanador-Castañeda, H.A., Estado actual de las competencias TIC en docentes, Puente, Revista Científica 2, 2015. [en línea]. Tomado de: puente.upbbga.edu.co/index.php/revistapuente/article/view/201/151, pp. $23-32,2015$.

[18] Sunkel, G., Trucco, D. y Móller, S., Aprender a enseñar con las tecnologias de la información y de la comunicación en América Latina: Potenciales beneficios, CEPAL. Proyecto financiado por la Unión Europea, Santiago de Chile, 2011.

[19] Acuña, A., Línea de investigación en tecnologías de la educación, Pontificia Universidad.[en línea]. 2016. Disponible en: http://www.javeriana.edu.co/Facultades/Educacion/08/encuentroEB/do cumentos/ponencia-1.pdf citado octubre de 2016, Bucaramanga, 2015.

[20] Hernández, Pausada, Gómez y Zúñiga, TIC y las personas de edad: Más allá de la usabilidad gerontología educativa, España, 2009.

[21] Sánchez-Llábana, J., Integración curricular de las TIC conceptos e ideas, Chile: Universidad de Chile, 2002.

[22] Sanchez, J., Integración curricular de TIC's conceptos y modelos, Enfoques Educacionales, pp. 51-70, 2003.

[23] Gusman-Acuña, J., Estudiantes universitarios: Entre la brecha digital y el aprendizaje. Apertura, [en línea]. 8(8), pp. 21-33, 2008. Disponible en: http://www.redalyc.org/articulo.oa?id=68811215002.

[24] Cabero-Almenara, J., Las necesidades de las TIC en el ámbito educativo: Oportunidades, riesgos y necesidades, Tecnología y Comunicación Educativas, pp. 1-16, 2007.

C.M. Duran-Chinchilla, recibe el título de Lic. en Lingüística y Literatura en 1997, en la Universidad de Pamplona, Colombia, el título de Esp. en Práctica Docente Universitaria en 2010, en la Universidad Francisco de Paula Santander Ocaña, Colombia; MSc. en Pedagogía, Universidad Francisco de Paula Santander Cúcuta, Colombia; Dr. en Ciencias de la Educación Universidad Pedagógica los Libertadores Venezuela (defensa de tesis septiembre de 2106 grado marzo de 2017). Sus intereses investigativos están direccionados a el área pedagógica y educativa, procesos de comunicación y TIC en procesos pedagógicos. Actualmente es docente ocasional del Departamento de Humanidades, Facultad de Educación Artes y Humanidades de la Universidad Francisco de Paula Santander, Ocaña, Colombia.

ORCID: 0000-0001-9291-7841

A.A. Rosado-Gomez, recibe el titulo Ing. de Sistemas en 2002, en la Univrsidad Francisco de Paula Santander, Cucuta, Colombia, el título de Esp. en Gestión de Proyectos Informáticos en 1998, en la Universidad de Pamplona, Pamplona, Colombia, el título de MSc.en Gestión Aplicación y Desarrollo de Software en 2013, en la Universidad Autónoma de Bucaramanga, Bucaramanga, Colombia. Sus intereses investigativos incluyen: la ingeniería de software, la minería de datos y elementos innovadores en la educación. Actualmente es el director de Departamento de Sistemas e Informatica de la Facultad de Ingenierias, Universidad Francisco de Paula Santander, Ocaña, Colombia.

ORCID: 0000-0003-2932-3383 